\title{
Coupled coincidence point technique and its application for solving nonlinear integral equations in RPOCbML spaces
}

\author{
Hasanen A. Hammad ${ }^{1 *}$ (D), Dhekra M. Albaqeri² and Rashwan A. Rashwan ${ }^{3}$
}

*Correspondence:

helmagd89@yahoo.com

1 Department of Mathematics,

Faculty of Science, Sohag

University, Sohag 82524, Egypt

Full list of author information is

available at the end of the article
The Author(s) 2019 Open Access This article is distributed under the terms of the Creative Commons Attribution 4.0 International License (http://creativecommons.org/licenses/by/4.0/), which permits unrestricted use, distribution, and reproduction in any medium, provided you give appropriate credit to the original author(s) and the source, provide a link to the Creative Commons license, and indicate if changes were made.

\section{Abstract}

The aim of this paper is to obtain some new important consequences related to coupled coincidence points via C-class functions in the context of a regular partial ordered complete $b$-metric-like space (for short, RPOCbML space); this space arises from combining the results of $b$-metric-like space with partial metric space and adding the regularity condition. Finally, we support our theoretical results by some examples and an application about finding an analytical solution for nonlinear integral equations.

Keywords: Coupled coincidence point, Metric-like spaces, Regular property, Nonlinear integral equations

2010 Mathematics Subject Classification: 46N40, 47H10, 46T99

\section{Introduction and elementary discussions}

Fixed point theory is one of the most important branches of non-linear analysis because it contributes to many disciplines such as economics (maximization and minimization problems), engineering and game theory, mathematics, and others. One of the most important advances in mathematics, for example, is to find solutions for linear and nonlinear systems and others. Due to the ease of this method, it became the most prevalent in functional analysis. This style emerged in 1922 when Banach [1] introduced his wellknown principle and called it Banach contraction principle in metric space. In 1992, Matthews [2] circulated the principle of Banach to partial metric space as follows:

Definition 1 [2] A mapping $p: \Upsilon \times \Upsilon \rightarrow \mathbb{R}^{+}$, where $\Upsilon$ is a nonempty set, is said to be a partial metric on $\Upsilon$ iffor any a,c,e $\in \Upsilon$ the following hypotheses hold:

$\left(P_{1}\right) a=c$ if and only if $p(a, a)=p(c, c)=p(a, c)$;

$\left(P_{2}\right) p(a, a) \leq p(a, c)$

$\left(P_{3}\right) p(a, c)=p(c, a)$

$\left(P_{4}\right) p(a, e) \leq p(a, c)+p(c, e)-p(c, c)$.

Then the pair $(\Upsilon, p)$ is called a partial metric space.

The concept of $b$-metric space was illustrated by Bakhtin [3] in 1993 and Czerwik [4] in 1998. Since then, many papers have been published on the fixed point theory of various 
classes of single-valued and multi-valued operators in $b$-metric spaces (see, [5-8] and references therein).

Definition 2 [4] Let $\Upsilon \neq \emptyset$ be a set and $s \geq 1$ be a given real number. A function $\omega_{b}: \Upsilon \times \Upsilon \rightarrow[0, \infty)$ is a b-metric if and only if, for all $a, c, e \in \Upsilon$, the following conditions are satisfied:

$\left(b_{1}\right) \omega_{b}(a, c)=0 \Leftrightarrow a=c$

$\left(b_{2}\right) \omega_{b}(a, c)=\omega_{b}(c, a)$

$\left(b_{3}\right) \omega_{b}(a, c) \leq s\left[\omega_{b}(a, e)+\omega_{b}(e, c)\right]$.

The pair $\left(\Upsilon, \omega_{b}\right)$ is called a b-metric space (with a coefficient $s \geq 1$ ).

Amini-Harandi [9] introduced the notion of metric-like spaces which is an interesting generalization of the spaces defined in [2] as follows:

Definition 3 [9] Let $\Upsilon$ be a nonempty set, a mapping $\omega: \Upsilon \times \Upsilon \rightarrow \mathbb{R}^{+}$is said to be a metric-like mapping on $\Upsilon$ if for all a, $c, e \in \Upsilon$ the following three conditions hold:

$\left(\omega_{1}\right) \omega(a, c)=0 \Rightarrow a=c$;

$\left(\omega_{2}\right) \omega(a, c)=\omega(c, a)$

$\left(\omega_{3}\right) \omega(a, e) \leq \omega(a, c)+\omega(c, e)$.

Then, the pair $(\Upsilon, \omega)$ is called a metric-like space.

A metric-like mapping on $\Upsilon$ satisfies all of the conditions of a metric except that $\omega(a, a)$ may be positive for $a \in \Upsilon$. Every partial metric space is a metric-like space but not conversely in general. For more details, see [9].

By considering the results of $b$-metric and metric-like spaces, the notion of $b$-metric-like space was presented by Alghamdi et al. [10]. They discussed some related fixed point consequences concerning with this space. Recently, Hussain et al. [11] examined topological structure of this space and presented some fixed point results in $b$-metric-like space. A lot of results on fixed points of mappings via certain contractive conditions in the mentioned spaces have been done (for example, see [11-13]).

Definition 4 [10] Let $\Upsilon \neq \emptyset$ be a set and $s \geq 1$ be a given real number. A function $\omega_{b}: \Upsilon \times \Upsilon \rightarrow[0, \infty)$ is a b-metric-like space if and only if, for all $a, c, e \in \Upsilon$, the following conditions are realized:

$\left(b_{1}\right) \omega_{b}(a, c)=0 \Rightarrow a=c$;

$\left(b_{2}\right) \omega_{b}(a, c)=\omega_{b}(c, a)$

$\left(b_{3}\right) \omega_{b}(a, c) \leq s\left[\omega_{b}(a, e)+\omega_{b}(e, c)\right]$.

The pair $\left(\Upsilon, \omega_{b}\right)$ is called a b-metric-like space (with a coefficient $s \geq 1$ ).

Example 1 Let $\Upsilon=[0, \infty)$. Define the function $\omega_{b}: \Upsilon^{2} \rightarrow[0, \infty)$ by $\omega_{b}(a, c)=(a+c)^{2}$. Then, $\left(\Upsilon, \omega_{b}\right)$ is a b-metric-like space with constant $s=2$, but it neither be a b-metric nor a metric-like space (see, [8]).

For more new examples related with metric-like and $b$-metric-like spaces, see $[14,15]$. 
Definition 5 [10] Let $\left(\Upsilon, \omega_{b}\right)$ be a b-metric-like space and $\left\{a_{n}\right\}$ be a sequence of points on $\Upsilon$. A point $a \in \Upsilon$ is called the limit of the sequence $\left\{a_{n}\right\}$ if $\lim _{n \rightarrow \infty} \omega_{b}\left(a, a_{n}\right)=\omega_{b}(a, a)$, and we say that the sequence $\left\{a_{n}\right\}$ is convergent to $a$ and denote it by $a_{n} \rightarrow a$ as $n \rightarrow \infty$.

Definition 6 [10] Let $\left(\Upsilon, \omega_{b}\right)$ be a b-metric-like space.

(i) A sequence $\left\{a_{n}\right\}$ is called Cauchy if and only if $\lim _{m, n \rightarrow \infty} \omega_{b}\left(a_{m}, a_{n}\right)$ exists and is finite.

(ii) A b-metric-like space $\left(X, \omega_{b}\right)$ is said to be complete if and only if every Cauchy sequence $\left\{a_{n}\right\}$ in $\Upsilon$ converges to $a \in \Upsilon$ such that $\lim _{m, n \rightarrow \infty} \omega_{b}\left(a_{m}, a_{n}\right)=\omega_{b}(a, a)=$ $\lim _{n \rightarrow \infty} \omega_{b}\left(a_{n}, a\right)$.

Lemma 1 [10] Let $\left\{c_{n}\right\}$ be a sequence in a b-metric-like space $\left(\Upsilon, \omega_{b}\right)$ such that

$$
\omega_{b}\left(c_{n}, c_{n+1}\right) \leq \lambda \omega_{b}\left(c_{n-1}, c_{n}\right)
$$

for some $\lambda \in\left(0, \frac{1}{s}\right)$, and for all $n \in \mathbb{N}$. Then, $\lim _{n \rightarrow \infty} \omega_{b}\left(c_{m}, c_{n}\right)=0$.

Let $\Upsilon \neq \emptyset$. Then, $\left(\Upsilon, \omega_{b}, \preceq\right)$ is called a partially ordered $b$-metric-like space if $\omega_{b}$ is a $b$ metric-like space on a partially ordered set $(\Upsilon, \preceq)$. Further, $\left(\Upsilon, \omega_{b}, \preceq\right)$ is called a regular partially ordered $b$-metric-like space if it has the following property:

(i) if a non - decreasing sequence $\left\{a_{n}\right\} \rightarrow a$, then $a_{n} \preceq a$, for all $n$,

(ii) if a non - increasing sequence $\left\{c_{n}\right\} \rightarrow c$, then $c \preceq c_{n}$ for all $n$.

In 1987, Guo and Lakshmikantham [16] first studied the concept of coupled fixed points. Later on, Bhaskar and Lakshmikantham [17] studied monotone property and supported this by providing an application to the existence of periodic boundary value problems. The obtained results were further extended by Lakshmikantham and Ćirić [18] to introducing the notion of a coupled coincidence point and coupled fixed point of mappings in partially ordered metric spaces.

Definition 7 [17] An element $(a, c) \in \Upsilon \times \Upsilon$ is called a coupled fixed point for the mapping $\Xi: \Upsilon \times \Upsilon \rightarrow \Upsilon$ if $\Xi(a, c)=a$ and $\Xi(c, a)=c$.

Definition 8 [17] An element $(a, c) \in \Upsilon \times \Upsilon$ is called a coupled coincidence point of the mappings $\Xi: \Upsilon \times \Upsilon \rightarrow \Upsilon$ and $\Theta: \Upsilon \rightarrow \Upsilon$ if $\Xi(a, c)=\Theta a$ and $\Xi(c, a)=\Theta c$.

Definition 9 [19] An element $(a, c) \in \Upsilon \times \Upsilon$ is called a coupled coincidence point of mappings $\Xi, \Theta: \Upsilon \times \Upsilon \rightarrow \Upsilon$ if $\Xi(a, c)=\Theta(a, c)$ and $\Xi(c, a)=\Theta(c, a)$.

Example 2 Let $\Xi, \Theta: \mathbb{R} \times \mathbb{R} \rightarrow \mathbb{R}$ be defined by $\Xi(a, c)=$ ac and $\Theta(a, c)=\frac{2}{3}(a+c)$ for all $(a, c) \in \Upsilon \times \Upsilon$. It is clear that $(0,0),(1,2)$, and $(2,1)$ are coupled coincidence points of non-self mappings $\Xi$ and $\Theta$.

Definition 10 [19] Suppose that $\Xi, \Theta: \Upsilon \times \Upsilon \rightarrow \Upsilon$ are two mappings. $\Xi$ is said to be $\Theta$-increasing with respect to $\preceq$ iffor all $a, c, l, m \in \Upsilon$, with $\Theta(a, c) \preceq \Theta(l, m)$ we have $\Xi(a, c) \preceq \Xi(l, m)$. 
Example 3 Let $\Upsilon=(0, \infty)$ be endowed with the natural ordering of real numbers $\leq$. Define mappings $\Xi, \Theta: \Upsilon \times \Upsilon \rightarrow \Upsilon$ by $\Xi(a, c)=\ln (a+c)$ and $\Theta(a, c)=a+c$ for all $(a, c) \in \Upsilon \times \Upsilon$. Then, $\Xi$ is $\Theta$-increasing with respect to $\leq$.

Throughout this paper, we assume that $\Psi=\{\psi: \psi:[0, \infty) \rightarrow[0, \infty)$ is nondecreasing and lower semi-continuous function, $\psi(t)=0 \Leftrightarrow t=0\}$.

In 2014, Ansari [20] introduced the concept of C-class functions which cover a large class of contractive conditions.

Definition 11 [20] A mappingf $:[0, \infty)^{2} \rightarrow \mathbb{R}$ is called C-class function if it is continuous and satisfies the following conditions:

(1) $f(\delta, \kappa) \leq \delta$,

(2) $f(\delta, \kappa)=\delta$ implies that either $\delta=0$ or $\kappa=0$ for all $\delta, \kappa \in[0, \infty)$.

We denote $C$-class functions as $\mathrm{C}$.

Example 4 The following functions $\Xi:[0, \infty)^{2} \rightarrow \mathbb{R}$ are elements of $\complement$, for all $\delta, \kappa \in$ $[0, \infty)$ :

(1) $f(\delta, \kappa)=\delta-\kappa$;

(2) $f(\delta, \kappa)=\lambda \delta, 0<\lambda<1$;

(3) $f(\delta, \kappa)=\frac{\delta}{(1+\kappa)^{r}} ; r \in(0, \infty)$;

(4) $f(\delta, \kappa)=\frac{\log \left(\kappa+a^{\delta}\right)}{(1+\kappa)}, a>1$;

(5) $f(\delta, 1)=\frac{\ln \left(1+b^{\delta}\right)}{2}, b>e$;

(6) $f(\delta, \kappa)=\phi(s)$, where $\phi:[0, \infty) \rightarrow[0, \infty)$ is a upper semi-continuous function such that $\phi(0)=0$, and $\phi(\kappa)<\kappa$ for $\kappa>0$;

(7) $f(\delta, \kappa)=\delta \beta(\delta), \beta:[0,1) \rightarrow[0,1)$;

(8) $f(\delta, \kappa)=\delta-\phi(\delta)$, where $\phi:[0, \infty) \rightarrow[0, \infty)$ is a upper semi-continuous function such that $\phi(0)=0$;

(9) $f(\delta, \kappa)=\delta-\frac{\kappa}{1-\kappa}$;

(10) $f(\delta, \kappa)=\delta h(\delta, \kappa)$, where $h:[0,1) \times[0,1) \rightarrow[0,1)$ is a continuous function such that $h(\kappa, \delta)<1$ for all $\kappa, \delta>0$.

In this paper, we prove some new coincidence coupled point results for the mappings $\Xi, \Theta: \Upsilon \times \Upsilon \rightarrow \Upsilon$ by using the notion of $C$-class of functions in RPOCbML spaces. We present some examples and an application to find the existence of solution of nonlinear integral equation to support our work. Our contributions in this direction improve and extend several comparable results in the literature for $b$-metric-like spaces.

\section{Main results}

Theorem 1 Let $\left(\Upsilon, \preceq, \omega_{b}\right)$ be a RPOCbML space (with a coefficient $s>1$ ). Assume that $\Xi, \Theta: \Upsilon \times \Upsilon \rightarrow \Upsilon$ are two mappings such that the following conditions are satisfied:

(1) $\Xi(\Upsilon \times \Upsilon) \subseteq \Theta(\Upsilon \times \Upsilon)$.

(2) $\Theta(\Upsilon \times \Upsilon)$ is closed.

(3) $\Xi$ is $\Theta$-increasing with respect to $\preceq$.

(4) There exist two elements $a_{0}, c_{0} \in \Upsilon$, with $\Theta\left(a_{0}, c_{0}\right) \preceq \Xi\left(a_{0}, c_{0}\right)$ and $\Theta\left(c_{0}, a_{0}\right) \succeq$ $\Xi\left(c_{0}, a_{0}\right)$. Suppose that there exist $\psi \in \Psi, 0<L<1$ and $f \in \complement$ such that: 
$\psi\left(s^{\alpha} \omega_{b}(\Xi(a, c), \Xi(l, m))\right) \leq f\left(\psi\left(\max \left\{\omega_{b}(\Theta(a, c), \Theta(l, m)), \omega_{b}(\Theta(c, a), \Theta(m, l))\right\}\right)\right.$,

$\left.L \max \left\{\omega_{b}(\Theta(a, c), \Theta(l, m)), \omega_{b}(\Theta(c, a), \Theta(m, l))\right\}\right),(1)$

for all $a, c, l, m \in \Upsilon, \alpha>0$ with $\Theta(a, c) \preceq \Theta(l, m)$ and $\Theta(c, a) \succeq \Theta(m, l)$ or $\Theta(c, a) \preceq$ $\Theta(m, l)$ and $\Theta(a, c) \succeq \Theta(l, m)$. Then, $\Xi$ and $\Theta$ have a coupled coincidence point in $\Upsilon$.

Proof Let $a_{0}, c_{0} \in \Upsilon$ be an arbitrary with $\Theta\left(a_{0}, c_{0}\right) \preceq \Xi\left(a_{0}, c_{0}\right)$ and $\Xi\left(c_{0}, a_{0}\right) \preceq$ $\Theta\left(c_{0}, a_{0}\right)$. Since $\Xi(\Upsilon \times \Upsilon) \subseteq \Theta(\Upsilon \times \Upsilon)$, there exists $\left(a_{1}, c_{1}\right) \in \Upsilon \times \Upsilon$ such that $\Xi\left(a_{0}, c_{0}\right)=\Theta\left(a_{1}, c_{1}\right)$ and $\Xi\left(c_{0}, a_{0}\right)=\Theta\left(c_{1}, a_{1}\right)$. Continuing this process, we can construct two sequences $\left\{a_{n}\right\}$ and $\left\{c_{n}\right\}$ in $\Upsilon$ such that

$$
\Xi\left(a_{n}, c_{n}\right)=\Theta\left(a_{n+1}, c_{n+1}\right), \quad \Xi\left(c_{n}, a_{n}\right)=\Theta\left(c_{n+1}, a_{n+1}\right), \quad \forall n \in \mathbb{N} .
$$

Now, we shall prove by induction that for all $n \in \mathbb{N}$, we have

$$
\Theta\left(a_{n}, c_{n}\right) \preceq \Theta\left(a_{n+1}, c_{n+1}\right) \text { and } \Theta\left(c_{n+1}, a_{n+1}\right) \preceq \Theta\left(c_{n}, a_{n}\right) \text {. }
$$

Since $\Theta\left(a_{0}, c_{0}\right) \preceq \Xi\left(a_{0}, c_{0}\right)$ and $\Xi\left(c_{0}, a_{0}\right) \preceq \Theta\left(c_{0}, a_{0}\right)$ and since $\Xi\left(a_{0}, c_{0}\right)=\Theta\left(a_{1}, c_{1}\right)$ and $\Xi\left(c_{0}, a_{0}\right)=\Theta\left(c_{1}, a_{1}\right)$, we have $\Theta\left(a_{0}, c_{0}\right) \preceq \Theta\left(a_{1}, c_{1}\right)$ and $\Theta\left(c_{1}, a_{1}\right) \preceq \Theta\left(c_{0}, a_{0}\right)$. Thus, (2) holds for $n=0$. Suppose that (2) holds for some fixed $n \in \mathbb{N}$. Since $\Xi$ is $\Theta$-increasing with respect to $\preceq$, we have

$$
\Theta\left(a_{n+1}, c_{n+1}\right)=\Xi\left(a_{n}, c_{n}\right) \preceq \Xi\left(a_{n+1}, c_{n+1}\right)=\Theta\left(a_{n+2}, c_{n+2}\right),
$$

and

$$
\Theta\left(c_{n+2}, a_{n+2}\right)=\Xi\left(c_{n+1}, a_{n+1}\right) \preceq \Xi\left(c_{n}, a_{n}\right)=\Theta\left(c_{n+1}, a_{n+1}\right) .
$$

Thus, (2) holds for all $n \in \mathbb{N}$. Denote

$$
\eta_{n}=\max \left\{\omega_{b}\left(\Theta\left(a_{n}, c_{n}\right), \Theta\left(a_{n+1}, c_{n+1}\right)\right), \omega_{b}\left(\Theta\left(c_{n}, a_{n}\right), \Theta\left(c_{n+1}, a_{n+1}\right)\right)\right\} .
$$

If $\Theta\left(a_{n}, c_{n}\right)=\Theta\left(a_{n+1}, c_{n+1}\right)$, then $\left(a_{n}, c_{n}\right)$ is a coincidence point and the proof is finished. So, we consider $\Theta\left(a_{n}, c_{n}\right) \neq \Theta\left(a_{n+1}, c_{n+1}\right)$ for all $n \in \mathbb{N}$, and we claim that

$$
\psi\left(s^{\alpha} \eta_{n+1}\right) \leq \psi\left(\eta_{n}\right)
$$

Since $\Theta\left(a_{n}, c_{n}\right) \preceq \Theta\left(a_{n+1}, c_{n+1}\right)$ and $\Theta\left(c_{n}, a_{n}\right) \succeq \Theta\left(c_{n+1}, a_{n+1}\right)$, using $a=a_{n}, c=$ $c_{n}, l=a_{n+1}$, and $m=c_{n+1}$ in (1), we get

$$
\begin{array}{r}
\psi\left(s^{\alpha} \omega_{b}\left(\Theta\left(a_{n+1}, c_{n+1}\right), \Theta\left(a_{n+2}, c_{n+2}\right)\right)\right)=\psi\left(s^{\alpha} \omega_{b}\left(\Xi\left(a_{n}, c_{n}\right), \Xi\left(a_{n+1}, c_{n+1}\right)\right)\right) \\
\leq f\left(\psi\left(\max \left\{\omega_{b}\left(\Theta\left(a_{n}, c_{n}\right), \Theta\left(a_{n+1}, c_{n+1}\right)\right), \omega_{b}\left(\Theta\left(c_{n}, a_{n}\right), \Theta\left(c_{n+1}, a_{n+1}\right)\right)\right\}\right),\right. \\
\left.L \max \left\{\omega_{b}\left(\Theta\left(a_{n}, c_{n}\right), \Theta\left(a_{n+1}, c_{n+1}\right)\right), \omega_{b}\left(\Theta\left(c_{n}, a_{n}\right), \Theta\left(c_{n+1}, a_{n+1}\right)\right)\right\}\right) .
\end{array}
$$

Similarly, we can write

$$
\begin{array}{r}
\psi\left(s^{\alpha} \omega_{b}\left(\Theta\left(c_{n+2}, a_{n+2}\right), \Theta\left(c_{n+1}, a_{n+1}\right)\right)\right)=\psi\left(s^{\alpha} \omega_{b}\left(\Theta\left(c_{n+1}, a_{n+1}\right), \Theta\left(c_{n}, a_{n}\right)\right)\right) \\
\leq f\left(\psi\left(\max \left\{\omega_{b}\left(\Theta\left(c_{n+1}, a_{n+1}\right), \Theta\left(c_{n}, a_{n}\right)\right), \omega_{b}\left(\Theta\left(a_{n+1}, c_{n+1}\right), \Theta\left(a_{n}, c_{n}\right)\right)\right\}\right),\right. \\
\left.L \max \left\{\omega_{b}\left(\Theta\left(c_{n+1}, a_{n+1}\right), \Theta\left(c_{n}, a_{n}\right)\right), \omega_{b}\left(\Theta\left(a_{n+1}, c_{n+1}\right), \Theta\left(a_{n}, c_{n}\right)\right)\right\}\right) .
\end{array}
$$

From (3) and (4), since $\psi$ is non-decreasing, we obtain that

$$
\begin{aligned}
& \psi\left(\max \left\{s^{\alpha} \omega_{b}\left(\Theta\left(a_{n+1}, c_{n+1}\right), \Theta\left(a_{n+2}, c_{n+2}\right)\right), s^{\alpha} \omega_{b}\left(\Theta\left(c_{n+2}, a_{n+2}\right), \Theta\left(c_{n+1}, a_{n+1}\right)\right)\right\}\right), \\
= & \max \left\{\psi\left(s^{\alpha} \omega_{b}\left(\Theta\left(a_{n+1}, c_{n+1}\right), \Theta\left(a_{n+2}, c_{n+2}\right)\right)\right), \psi\left(s^{\alpha} \omega_{b}\left(\Theta\left(c_{n+2}, a_{n+2}\right), \Theta\left(c_{n+1}, a_{n+1}\right)\right)\right)\right\}, \\
\leq & f\left(\psi\left(\max \left\{\omega_{b}\left(\Theta\left(a_{n}, c_{n}\right), \Theta\left(a_{n+1}, c_{n+1}\right)\right), \omega_{b}\left(\Theta\left(c_{n}, a_{n}\right), \Theta\left(c_{n+1}, a_{n+1}\right)\right)\right\}\right),\right. \\
& \left.L \max \left\{\omega_{b}\left(\Theta\left(a_{n}, c_{n}\right), \Theta\left(a_{n+1}, c_{n+1}\right)\right), \omega_{b}\left(\Theta\left(c_{n}, a_{n}\right), \Theta\left(c_{n+1}, a_{n+1}\right)\right)\right\}\right) .
\end{aligned}
$$


This means

$$
\psi\left(s^{\alpha} \eta_{n+1}\right) \leq f\left(\psi\left(\eta_{n}\right), L \eta_{n}\right) \leq \psi\left(\eta_{n}\right) .
$$

Since the function $\psi$ is non-decreasing, so the inequality (5) implies that

$$
\eta_{n+1} \leq \frac{1}{s^{\alpha}} \eta_{n}
$$

Therefore, by Lemma (1), we have $\left\{\eta_{n}\right\}$ is a Cauchy sequence. Since

$$
\eta_{n}=\max \left\{\omega_{b}\left(\Theta\left(a_{n}, c_{n}\right), \Theta\left(a_{n+1}, c_{n+1}\right)\right), \omega_{b}\left(\Theta\left(c_{n}, a_{n}\right), \Theta\left(c_{n+1}, a_{n+1}\right)\right)\right\},
$$

then both sequences $\left\{\Theta\left(a_{n}, c_{n}\right)\right\}$ and $\left\{\Theta\left(c_{n}, a_{n}\right)\right\}$ are Cauchy in the complete space $\left(\Upsilon, \omega_{b}\right)$, and since $\Xi(\Upsilon \times \Upsilon) \subseteq \Theta(\Upsilon \times \Upsilon)$ and $\Theta(\Upsilon \times \Upsilon)$ is closed, there exist $a, c \in \Upsilon$ such that

$$
\lim _{n \rightarrow \infty} \Theta\left(a_{n}, c_{n}\right)=\Theta(a, c) \text { and } \lim _{n \rightarrow \infty} \Theta\left(c_{n}, a_{n}\right)=\Theta(c, a) .
$$

Correspondingly, we have

$$
\omega_{b}(\Theta(a, c), \Theta(a, c))=\lim _{n \rightarrow \infty} \omega_{b}\left(\Theta\left(a_{n}, c_{n}\right), \Theta(a, c)\right)=0,
$$

and

$$
\omega_{b}(\Theta(c, a), \Theta(c, a))=\lim _{n \rightarrow \infty} \omega_{b}\left(\Theta\left(c_{n}, a_{n}\right), \Theta(c, a)\right)=0 .
$$

By the regularity of the space $\left(\Upsilon, \omega_{b}, \preceq\right)$, we get

$$
\Theta\left(a_{n}, c_{n}\right) \preceq \Theta(a, c) \text { and } \Theta\left(c_{n}, a_{n}\right) \succeq \Theta(c, a) .
$$

It follows from (1) that

$$
\begin{aligned}
\psi\left(s^{\alpha} \omega_{b}\left(\Xi\left(a_{n}, c_{n}\right), \Xi(a, c)\right)\right) \leq & f\left(\psi\left(\max \left\{\omega_{b}\left(\Theta\left(a_{n}, c_{n}\right), \Theta(a, c)\right), \omega_{b}\left(\Theta\left(c_{n}, a_{n}\right), \Theta(c, a)\right)\right\}\right),\right. \\
& \left.L \max \left\{\omega_{b}\left(\Theta\left(a_{n}, c_{n}\right), \Theta(a, c)\right), \omega_{b}\left(\Theta\left(c_{n}, a_{n}\right), \Theta(c, a)\right)\right\}\right) .
\end{aligned}
$$

Passing the limit as $n \rightarrow+\infty$, using (6) and (7), we have

$$
\left.\lim _{n \rightarrow \infty} \omega_{b}\left(\Xi\left(a_{n}, c_{n}\right), \Xi(a, c)\right)\right)=0 \text { implies } \Xi\left(a_{n}, c_{n}\right)=\Xi(a, c) .
$$

Similarly, using (6) and (7), we obtain

$$
\Xi\left(c_{n}, a_{n}\right)=\Xi(c, a) .
$$

By triangle inequality in $b$-metric-like space, one can write

$$
\begin{aligned}
\omega_{b}(\Theta(a, c), \Xi(a, c)) & \leq s\left[\omega_{b}\left(\Theta(a, c), \Theta\left(a_{n+1}, c_{n+1}\right)\right)+\omega_{b}\left(\Theta\left(a_{n+1}, c_{n+1}\right), \Xi(a, c)\right)\right] \\
& =s\left[\omega_{b}\left(\Theta(a, c), \Theta\left(a_{n+1}, c_{n+1}\right)\right)+\omega_{b}\left(\Xi\left(a_{n}, c_{n}\right), \Xi(a, c)\right)\right] .
\end{aligned}
$$

Taking the limit as $n \rightarrow \infty$ and using (6) and (8), we have

$$
\omega_{b}(\Theta(a, c), \Xi(a, c))=0 \text { implies } \Theta(a, c)=\Xi(a, c) .
$$

Similarly, by (7) and (9), we can show that

$$
\omega_{b}(\Theta(c, a), \Xi(c, a))=0 \text { implies } \Theta(c, a)=\Xi(c, a) .
$$

Thus, $\Xi$ and $\Theta$ have a coupled coincidence point.

To prove the uniqueness of a coupled coincidence point, define the partial ordered in $(\Upsilon \times \Upsilon, \preceq)$ by for all $(a, c),(l, m) \in \Upsilon \times \Upsilon,(a, c) \preceq(l, m)$ if and only if $\Theta(a, c) \preceq \Theta(l, m)$ and $\Theta(c, a) \succeq \Theta(m, l)$ where $\Theta: \Upsilon \times \Upsilon \rightarrow \Upsilon$ is one-one. 
Theorem 2 In addition to the hypotheses of Theorem 1, suppose that for every $(a, c),\left(a^{*}, c^{*}\right)$ in $\Upsilon \times \Upsilon$, there exists another $(l, m)$ in $\Upsilon \times \Upsilon$ which is comparable to $(a, c)$ and $\left(a^{*}, c^{*}\right)$, then $\Xi$ and $\Theta$ have a unique coupled coincidence point.

Proof From Theorem 1, we know that the set of coupled coincidence points of $\Xi$ and $\Theta$ is nonempty. Suppose $(a, c)$ and $\left(a^{*}, c^{*}\right)$ are coupled coincidence points of $\Xi$ and $\Theta$ that is

$$
\Xi(a, c)=\Theta(a, c), \quad \Xi(c, a)=\Theta(c, a),
$$

and

$$
\Xi\left(a^{*}, c^{*}\right)=\Theta\left(a^{*}, c^{*}\right), \quad \Xi\left(c^{*}, a^{*}\right)=\Theta\left(c^{*}, a^{*}\right) .
$$

Now we prove that $\Theta(a, c)=\Theta\left(a^{*}, c^{*}\right)$ and $\Theta(c, a)=\Theta\left(c^{*}, a^{*}\right)$. By assumption, there exists $(l, m)$ in $\Upsilon \times \Upsilon$ that is comparable to $(a, c)$ and $\left(a^{*}, c^{*}\right)$. We define sequences $\left\{\Theta\left(l_{n}, m_{n}\right)\right\}$ and $\left\{\Theta\left(m_{n}, l_{n}\right)\right\}$ as follows:

$$
l_{0}=l, m_{0}=m, \Xi\left(l_{n}, m_{n}\right)=\Theta\left(l_{n+1}, m_{n+1}\right), \Xi\left(m_{n}, l_{n}\right)=\Theta\left(m_{n+1}, l_{n+1}\right), \forall n \in \mathbb{N} .
$$

Since $(l, m)$ is comparable to $(a, c)$, we assume that $(a, c) \preceq(l, m)=\left(l_{0}, m_{0}\right)$. This implies $\Theta(a, c) \preceq \Theta\left(l_{0}, m_{0}\right)$ and $\Theta(c, a) \succeq \Theta\left(m_{0}, l_{0}\right)$. Suppose that $(a, c) \preceq\left(l_{n}, m_{n}\right)$ for some $n$. We claim that

$$
(a, c) \preceq\left(l_{n+1}, m_{n+1}\right) .
$$

Since $\Xi$ is $\Theta$ increasing, then $\Theta(a, c) \preceq \Theta\left(l_{n}, m_{n}\right)$ implies $\Xi(a, c) \preceq \Xi\left(l_{n}, m_{n}\right)$ and $\Theta(c, a) \succeq \Theta\left(m_{n}, l_{n}\right)$ implies $\Xi(c, a) \succeq \Xi\left(m_{n}, l_{n}\right)$.

Thus,

$$
\Theta(a, c)=\Xi(a, c) \preceq \Xi\left(l_{n}, m_{n}\right)=\Theta\left(l_{n+1}, m_{n+1}\right),
$$

and

$$
\Theta(c, a)=\Xi(c, a) \succeq \Xi\left(m_{n}, l_{n}\right)=\Theta\left(m_{n+1}, l_{n+1}\right) .
$$

It follows that

$$
(a, c) \preceq\left(l_{n}, m_{n}\right), \forall n \text {. }
$$

Using (1) and (10), we have

$$
\begin{array}{r}
\psi\left(s^{\alpha} \omega_{b}\left(\Theta(a, c), \Theta\left(l_{n+1}, m_{n+1}\right)\right)\right)=\psi\left(s^{\alpha} \omega_{b}\left(\Xi(a, c), \Xi\left(l_{n}, m_{n}\right)\right)\right) \\
\leq f\left(\psi\left(\max \left\{\omega_{b}\left(\Theta(a, c), \Theta\left(l_{n}, m_{n}\right)\right), \omega_{b}\left(\Theta(c, a), \Theta\left(m_{n}, l_{n}\right)\right)\right\}\right),\right. \\
\left.L \max \left\{\omega_{b}\left(\Theta(a, c), \Theta\left(l_{n}, m_{n}\right)\right), \omega_{b}\left(\Theta(c, a), \Theta\left(m_{n}, l_{n}\right)\right)\right\}\right) .
\end{array}
$$

Similarly,

$$
\begin{array}{r}
\psi\left(s^{\alpha} \omega_{b}\left(\Theta\left(m_{n+1}, l_{n+1}\right), \Theta(c, a)\right)\right)=\psi\left(s^{\alpha} \omega_{b}\left(\Xi\left(m_{n}, l_{n}\right), \Xi(c, a)\right)\right) \\
\leq f\left(\psi\left(\max \left\{\omega_{b}\left(\Theta\left(m_{n}, l_{n}\right), \Theta(c, a)\right), \omega_{b}\left(\Theta\left(l_{n}, m_{n}\right), \Theta(a, c)\right)\right\}\right),\right. \\
\left.L \max \left\{\omega_{b}\left(\Theta\left(m_{n}, l_{n}\right), \Theta(c, a)\right), \omega_{b}\left(\Theta\left(l_{n}, m_{n}\right), \Theta(a, c)\right)\right\}\right) .
\end{array}
$$

Using (11), (12) with the property of $f$ and since $\psi$ is nondecreasing, we have

$$
\begin{array}{r}
\psi\left(\max \left\{s^{\alpha} \omega_{b}\left(\Theta(a, c), \Theta\left(l_{n+1}, m_{n+1}\right)\right), s^{\alpha} \omega_{b}\left(\Theta\left(m_{n+1}, l_{n+1}\right), \Theta(c, a)\right)\right\}\right) \\
\leq f\left(\psi\left(\max \left\{\omega_{b}\left(\Theta(a, c), \Theta\left(l_{n}, m_{n}\right)\right), \omega_{b}\left(\Theta(c, a), \Theta\left(m_{n}, l_{n}\right)\right)\right\}\right),\right. \\
\left.L \max \left\{\omega_{b}\left(\Theta(a, c), \Theta\left(l_{n}, m_{n}\right)\right), \omega_{b}\left(\Theta(c, a), \Theta\left(m_{n}, l_{n}\right)\right)\right\}\right) \\
\leq \psi\left(\max \left\{\omega_{b}\left(\Theta(a, c), \Theta\left(l_{n}, m_{n}\right)\right), \omega_{b}\left(\Theta(c, a), \Theta\left(m_{n}, l_{n}\right)\right)\right\}\right) .
\end{array}
$$


Using the property of $\psi$ in (13), we get

$$
\begin{array}{r}
\max \left\{\omega_{b}\left(\Theta(a, c), \Theta\left(l_{n+1}, m_{n+1}\right)\right), \omega_{b}\left(\Theta\left(m_{n+1}, l_{n+1}\right), \Theta(c, a)\right)\right\} \\
\leq \frac{1}{s^{\alpha}} \max \left\{\omega_{b}\left(\Theta(a, c), \Theta\left(l_{n}, m_{n}\right)\right), \omega_{b}\left(\Theta(c, a), \Theta\left(m_{n}, l_{n}\right)\right)\right\} .
\end{array}
$$

It follows that $\max \left\{\omega_{b}\left(\Theta(a, c), \Theta\left(l_{n}, m_{n}\right)\right), \omega_{b}\left(\Theta(c, a), \Theta\left(m_{n}, l_{n}\right)\right)\right\}$ is nonnegative decreasing sequence and

$$
\lim _{n \rightarrow \infty} \max \left\{\omega_{b}\left(\Theta(a, c), \Theta\left(l_{n}, m_{n}\right)\right), \omega_{b}\left(\Theta(c, a), \Theta\left(m_{n}, l_{n}\right)\right)\right\}=0,
$$

which implies that

$$
\lim _{n \rightarrow \infty} \omega_{b}\left(\Theta(a, c), \Theta\left(l_{n}, m_{n}\right)\right)=\lim _{n \rightarrow \infty} \omega_{b}\left(\Theta(c, a), \Theta\left(m_{n}, l_{n}\right)\right)=0 .
$$

Repeating the same process, we can prove that

$$
\lim _{n \rightarrow \infty} \omega_{b}\left(\Theta\left(a^{*}, c^{*}\right), \Theta\left(l_{n}, m_{n}\right)\right)=\lim _{n \rightarrow \infty} \omega_{b}\left(\Theta\left(c^{*}, a^{*}\right), \Theta\left(m_{n}, l_{n}\right)\right)=0 .
$$

By triangle inequality in b-metric-like space, we have

$$
\omega_{b}\left(\Theta(a, c), \Theta\left(a^{*}, c^{*}\right)\right) \leq s\left[\omega_{b}\left(\Theta(a, c), \Theta\left(l_{n}, m_{n}\right)\right)+\omega_{b}\left(\Theta\left(a^{*}, c^{*}\right), \Theta\left(l_{n}, m_{n}\right)\right)\right],
$$

and

$$
\omega_{b}\left(\Theta(c, a), \Theta\left(c^{*}, a^{*}\right)\right) \leq s\left[\omega_{b}\left(\Theta(c, a), \Theta\left(m_{n}, l_{n}\right)\right)+\omega_{b}\left(\Theta\left(c^{*}, a^{*}\right), \Theta\left(m_{n}, l_{n}\right)\right)\right] .
$$

Passing the limit as $n \rightarrow \infty$ into the above inequalities, using (14) and (15), we get $\Theta(a, c)=\Theta\left(a^{*}, c^{*}\right)$ and $\Theta(c, a)=\Theta\left(c^{*}, a^{*}\right)$.

The following examples justify all requirements of the hypotheses of Theorem 1 .

Example 5 Let $f(\delta, \kappa)=\tau \delta, \Upsilon=[0, \infty)$ endowed with the natural ordering of real numbers. We endow $\Upsilon$ with

$$
\omega_{b}(a, c)=(\max \{a, c\})^{2}
$$

for all $a, c \in \Upsilon$. Then, $\left(\Upsilon, \omega_{b}\right)$ is a complete $b$-metric-like space with a coefficient $s=2$. Let the sequence $\left\{a_{n}\right\}$ of monotone non-decreasing in $\Upsilon$ such that $\lim _{n \rightarrow \infty} a_{n}=a \in \Upsilon$, then the sequences (sequences of real numbers)

$$
a_{1}(t) \leq a_{2}(t) \leq \ldots \leq a_{n}(t) \leq \ldots
$$

converge also to $a(t)$ for all $t \in[0, \infty)$. So $a_{n}(t) \leq a(t)$ for all $t \in \Upsilon, n \in \mathbb{N}$. Therefore, $a_{n} \preceq a$ for all $n$. By the same manner, one can show that the monotone non-increasing sequence $\left\{c_{n}\right\}$ in $\Upsilon$ such that $\lim _{n \rightarrow \infty} c_{n}=c \in \Upsilon$ is a lower bound for all the elements in the sequence, i.e., $c \preceq c_{n}$ for all $n$. Therefore, the regularity condition hold.

Also, if we define an order relation on $\Upsilon$ as $a \preceq c$ for all $a, c \in \Upsilon$, we conclude that $\Upsilon$ is a partially ordered set, so we deduce that $\left(\Upsilon, \omega_{b}, \preceq\right)$ is a RPOCbML space.

Define mappings $\Xi, \Theta: \Upsilon \times \Upsilon \rightarrow \Upsilon$ as follows:

$$
\Xi(a, c)=\left\{\begin{array}{c}
\frac{a-c}{4}, \quad a \geq c \\
0, \quad a<c
\end{array} \text { and } \Theta(a, c)=\left\{\begin{array}{cc}
a-c, & a \geq c \\
0, & a<c
\end{array},\right.\right.
$$

for all a, $c \in \Upsilon$. It is clear that $\Xi(\Upsilon, \Upsilon) \subseteq \Theta(\Upsilon, \Upsilon)$ and $\Theta(\Upsilon, \Upsilon)$ is closed.

Now, we are going to prove that $\Xi$ is $\Theta$-increasing. Suppose that $(a, c),(l, m) \in \Upsilon \times \Upsilon$ with $\Theta(a, c) \leq \Theta(l, m)$, we state the following cases:

Case 1. If $a<c$, then $\Xi(a, c)=0 \leq \Theta(a, c)$. 
Case 2. If $a \geq c$ and $l \geq m$, then $\Theta(a, c) \leq \Theta(l, m)$ leads to $a-c \leq l-m$, so

$$
\frac{a-c}{4} \leq \frac{l-m}{4} \text { implies } \Xi(a, c) \leq \Xi(l, m)
$$

Case 3. If $l<m$, then $\Theta(a, c) \leq \Theta(l, m)$ implies that $0 \leq a-c \leq 0$, hence $a=c$, so $\Xi(a, c)=0 \leq \Theta(a, c)$. From the above cases, we deduce that $\Xi$ is $\Theta$-increasing.

Next, we prove that there exist two elements $a_{\circ}, c_{\circ} \in \Upsilon$ with $\Theta\left(a_{\circ}, c_{\circ}\right) \preceq \Xi\left(a_{\circ}, c_{\circ}\right)$ and $\Theta\left(c_{\circ}, a_{\circ}\right) \succeq \Xi\left(c_{\circ}, a_{\circ}\right)$.

Since we get

$$
\Theta\left(0, \frac{1}{3}\right)=0=\Xi\left(0, \frac{1}{3}\right) \text { and } \Theta\left(\frac{1}{3}, 0\right)=\frac{1}{3}>\frac{1}{12}=\Xi\left(\frac{1}{3}, 0\right) .
$$

So the two elements are $0, \frac{1}{3} \in \Upsilon$. Now, define the function $\psi:[0, \infty) \rightarrow[0, \infty)$ as $\psi(\kappa)=$ $\frac{1}{4} \kappa$ for all $\kappa \in[0, \infty)$; it is obvious that $\psi \in \Psi$. Finally, we justify the contraction (1) for all $a, b, l, m \in \Upsilon$, with $\Theta(a, c) \leq \Theta(l, m)$ and $\Theta(c, a) \geq \Theta(m, l)$ or $\Theta(a, c) \geq \Theta(l, m)$ and $\Theta(c, a) \leq \Theta(m, l)$, we get

$$
\begin{aligned}
\psi\left(s^{\alpha}(\Xi(a, c), \Xi(l, m))\right) & =\frac{1}{2} 2^{2} \omega_{b}(\Xi(a, c), \Xi(l, m)) \\
& =2(\max \{\Xi(a, c), \Xi(l, m)\})^{2} \\
& =\frac{2}{16}(\max \{\Theta(a, c), \Theta(l, m)\})^{2} \\
& \leq \frac{1}{4} \omega_{b}(\Theta(a, c), \Theta(l, m)) \\
& \leq \frac{1}{4} \max \left\{\omega_{b}(\Theta(a, c), \Theta(l, m)), \omega_{b}(\Theta(c, a), \Theta(m, l))\right\} \\
& =\frac{1}{2} \psi\left(\max \left\{\omega_{b}(\Theta(a, c), \Theta(l, m)), \omega_{b}(\Theta(c, a), \Theta(m, l))\right\}\right) \\
& =m \psi\left(\max \left\{\omega_{b}(\Theta(a, c), \Theta(l, m)), \omega_{b}(\Theta(c, a), \Theta(m, l))\right\}\right) \\
& =f\left(\psi\left(\max \left\{\omega_{b}(\Theta(a, c), \Theta(l, m)), \omega_{b}(\Theta(c, a), \Theta(m, l))\right\}\right),\right),
\end{aligned}
$$

where $L \in(0,1), \alpha=2>0$, and $\tau=\frac{1}{2}$. Hence, the hypothesis (1) is satisfied. Thus, all requirements of the hypotheses of Theorem 1 hold and $(0,0)$ is a coupled coincidence point of $\Xi$ and $\Theta$ and in the same time is a coupled fixed point.

Example 6 Let $f(\delta, \kappa)=\delta-\kappa, \Upsilon=[0, \infty)$ endowed with the natural ordering of real numbers. We endow $\Upsilon$ with

$$
\omega_{b}(a, c)=a^{2}+c^{2}+|a-c|^{2}
$$

for all $a, c \in \Upsilon$. Then, $\left(\Upsilon, \preceq, \omega_{b}\right)$ is a RPOCbML space with a coefficient $s=2$ (as in the above example).

Define mappings $\Xi, \Theta: \Upsilon \times \Upsilon \rightarrow \Upsilon$ as follows:

$$
\Xi(a, c)=\left\{\begin{array}{cl}
\frac{1}{8} \ln \left(1+\frac{a-c}{2}\right), & a \geq c \\
0, & a<c
\end{array} \text { and } \Theta(a, c)=\left\{\begin{array}{cc}
\frac{a-c}{2}, & a \geq c \\
0, & a<c
\end{array},\right.\right.
$$

for all $a, b, l, m \in \Upsilon$. It is clear that $\Xi(\Upsilon, \Upsilon) \subseteq \Theta(\Upsilon, \Upsilon)$ and $\Theta(\Upsilon, \Upsilon)$ is closed.

Now, we should show that $\Xi$ is $\Theta$-increasing. Let $(a, c),(l, m) \in \Upsilon \times \Upsilon$ with $\Theta(a, c) \leq$ $\Theta(l, m)$, we state the following cases:

Case 1. If $a<c$, then $\Xi(a, c)=0 \leq \Theta(a, c)$. 
Case 2. If $a \geq c$ and $l \geq m$, then $\Theta(a, c) \leq \Theta(l, m)$, leads to $\frac{a-c}{2} \leq \frac{l-m}{2}$, so

$$
\frac{\ln \left(1+\frac{a-c}{2}\right)}{16} \leq \frac{\ln \left(1+\frac{l-m}{2}\right)}{16} \text { implies } \Xi(a, c) \leq \Xi(l, m) \text {. }
$$

Case 3. If $l<m$, then $\Theta(a, c) \leq \Theta(l, m)$ implies that

$$
0 \leq \frac{a-b}{2} \leq 0 \text { implies } a-b=0 \text { implies } a=b,
$$

so $\Xi(a, c)=0 \leq \Theta(a, c)$. From the three cases above, we deduce that $\Xi$ is $\Theta$-increasing.

Next, as in Example 5, we prove that there exist two elements $a_{\circ}, c_{\circ} \in \Upsilon$ with $\Theta\left(a_{\circ}, c_{\circ}\right) \preceq$ $\Xi\left(a_{\circ}, c_{\circ}\right)$ and $\Theta\left(a_{\circ}, c_{\circ}\right) \succeq \Theta\left(c_{\circ}, a_{\circ}\right)$. So, we get

$$
\Theta(0,1)=0=\Xi(0,1) \text { and } \Theta(1,0)=\frac{1}{2}>\frac{1}{8} \ln \left(\frac{3}{2}\right)=\Xi(1,0) .
$$

So the two elements are $0,1 \in \Upsilon$. Now, define the function $\psi:[0, \infty) \rightarrow[0, \infty)$ as $\psi(\kappa)=$ $\frac{1}{8} \kappa$ for all $\kappa \in[0, \infty)$; it is obvious that $\psi \in \Psi$. Finally, we verify the contraction (1) for all $a, c, l, m \in \Upsilon$, with $\Theta(a, c) \leq \Theta(l, m)$ and $\Theta(c, a) \geq \Theta(m, l)$ or $\Theta(a, c) \geq \Theta(l, m)$ and $\Theta(c, a) \leq \Theta(m, l)$. Since $\ln (1+\kappa) \leq \kappa$ for all $\kappa \in[0, \infty)$, then we have

$$
\begin{aligned}
& \psi\left(s^{\alpha}(\Xi(a, c), \Xi(l, m))\right)= \\
= & \frac{1}{8} 2^{2} \omega_{b}(\Xi(a, c), \Xi(l, m)) \\
= & \frac{1}{128}\left[\left(\ln \left(1+\frac{a-c}{2}\right)\right)^{2}+\left(\ln \left(1+\frac{l-m}{2}\right)\right)^{2}+\left|\ln \left(1+\frac{a-c}{2}\right)-\ln \left(1+\frac{l-m}{2}\right)\right|^{2}\right] \\
\leq & \frac{1}{128}\left[\left(\frac{a-c}{2}\right)^{2}+\left(\frac{l-m}{2}\right)^{2}+\left|\frac{a-c}{2}-\frac{l-m}{2}\right|^{2}\right] \\
\leq & \frac{1}{128} \omega_{b}(\Theta(a, c), \Theta(l, m)) \\
\leq & \frac{1}{128} \max \left\{\omega_{b}(\Theta(a, c), \Theta(l, m)), \omega_{b}(\Theta(c, a), \Theta(m, l))\right\} \\
= & \left(\frac{1}{8}-\frac{15}{128}\right) \max \left\{\omega_{b}(\Theta(a, c), \Theta(l, m)), \omega_{b}(\Theta(c, a), \Theta(m, l))\right\} \\
= & \psi\left(\max \left\{\omega_{b}(\Theta(a, c), \Theta(l, m)), \omega_{b}(\Theta(c, a), \Theta(m, l))\right\}\right) \\
& -L \max \left\{\omega_{b}(\Theta(a, c), \Theta(l, m)), \omega_{b}(\Theta(c, a), \Theta(m, l))\right\} \\
= & f\left(\begin{array}{c}
\left.\max \left\{\omega_{b}(\Theta(a, c), \Theta(l, m)), \omega_{b}(\Theta(c, a), \Theta(m, l))\right\}\right), \\
L \max \left\{\omega_{b}(\Theta(a, c), \Theta(l, m)), \omega_{b}(\Theta(c, a), \Theta(m, l))\right\}
\end{array}\right)
\end{aligned}
$$

where $L=\frac{15}{128}<1$ and $\alpha=2>0$. Hence, the condition (1) is satisfied. Thus, all requirements of the hypotheses of Theorem 1 are verified and $(0,0)$ is a coupled coincidence point of $\Xi$ and $\Theta$, and in the same time is a coupled fixed point.

\section{Consequences of the main results}

If we put $f(\delta, \kappa)=\delta-\kappa$ in Theorem 1 , we get the following corollary:

Corollary 1 Let $\left(\Upsilon, \preceq, \omega_{b}\right)$ be a RPOCbML space (with parameter $\left.s>1\right)$. Assume that $\Xi, \Theta: \Upsilon \times \Upsilon \rightarrow \Upsilon$ are two mappings such that the following conditions are satisfied:

(1) $\Xi(\Upsilon \times \Upsilon) \subseteq \Theta(\Upsilon \times \Upsilon)$.

(2) $\Theta(\Upsilon \times \Upsilon)$ is closed.

(3) $\Xi$ is $\Theta$-increasing with respect to $\preceq$.

(4) There exist two elements $a_{0}, c_{0} \in \Upsilon$, with $\Theta\left(a_{0}, c_{0}\right) \preceq \Xi\left(a_{0}, c_{0}\right)$ and $\Theta\left(c_{0}, a_{0}\right) \succeq$ $\Xi\left(c_{0}, a_{0}\right)$. Suppose that there exist $\psi \in \Psi, 0<L<1$ such that: 


$$
\begin{aligned}
\psi\left(s^{\alpha} \omega(\Xi(a, c), \Xi(l, m))\right) \leq & \psi\left(\max \left\{\omega_{b}(\Theta(a, c), \Theta(l, m)), \omega_{b}(\Theta(c, a), \Theta(m, l))\right\}\right) \\
& \left.\left.-L \max \left\{\omega_{b}(\Theta(a, c), \Theta(l, m)), \omega_{b}(\Theta(c, a)), \Theta(m, l)\right)\right)\right\}
\end{aligned}
$$

for all $a, c, l, m \in \Upsilon, \alpha>0$ with $\Theta(a, c) \preceq \Theta(l, m)$ and $\Theta(c, a) \succeq \Theta(m, l)$ or $\Theta(c, a) \preceq$ $\Theta(m, l)$ and $\Theta(a, c) \geq \Theta(l, m)$. Then, $\Xi$ and $\Theta$ have a coupled coincidence point in $\Upsilon$.

If we choose $f(\delta, \kappa)=k \delta, 0 \leq k<1$ in Theorem 1 , we get the following result:

Corollary 2 Let $\left(X, \preceq, \omega_{b}\right)$ be a RPOCbML space (with coefficient $s>1$ ). Assume that $F, G: X \times X \rightarrow X$ are two mappings such that the following conditions are realized:

(1) $\Xi(\Upsilon \times \Upsilon) \subseteq \Theta(\Upsilon \times \Upsilon)$.

(2) $\Theta(\Upsilon \times \Upsilon)$ is closed.

(3) $\Xi$ is $\Theta$-increasing with respect to $\preceq$.

(4) There exist two elements $a_{0}, c_{0} \in \Upsilon$, with $\Theta\left(a_{0}, c_{0}\right) \preceq \Xi\left(a_{0}, c_{0}\right)$ and $\Theta\left(c_{0}, a_{0}\right) \succeq$ $\Xi\left(c_{0}, a_{0}\right)$. Suppose that there exist $\psi \in \Psi$ such that:

$$
\psi\left(s^{\alpha} \omega(\Xi(a, c), \Xi(l, m))\right) \leq k \psi\left(\max \left\{\omega_{b}(\Theta(a, c), \Theta(l, m)), \omega_{b}(\Theta(c, a), \Theta(m, l))\right\}\right),
$$

for all $a, c, l, m \in \Upsilon, \alpha>0$ with $\Theta(a, c) \preceq \Theta(l, m)$ and $\Theta(c, a) \succeq \Theta(m, l)$ or $\Theta(c, a) \preceq$ $\Theta(m, l)$ and $\Theta(a, c) \geq \Theta(l, m)$. Then, $\Xi$ and $\Theta$ have a coupled coincidence point in $\Upsilon$.

If we take $\psi(t)=t$ in Theorem 1, we get the following consequence:

Corollary 3 Let $\left(X, \preceq, \omega_{b}\right)$ be a RPOCbML space (with coefficient $s>1$ ). Assume that $F, G: X \times X \rightarrow X$ are two mappings such that the following conditions hold:

(1) $\Xi(\Upsilon \times \Upsilon) \subseteq \Theta(\Upsilon \times \Upsilon)$.

(2) $\Theta(\Upsilon \times \Upsilon)$ is closed.

(3) $\Xi$ is $\Theta$-increasing with respect to $\preceq$.

(4) There exist two elements $a_{0}, c_{0} \in \Upsilon$, with $\Theta\left(a_{0}, c_{0}\right) \preceq \Xi\left(a_{0}, c_{0}\right)$ and $\Theta\left(c_{0}, a_{0}\right) \succeq$ $\Xi\left(c_{0}, a_{0}\right)$. Suppose that $0<L<1$ such that:

$$
\begin{aligned}
s^{\alpha} \omega(\Xi(a, c), \Xi(l, m)) \leq & f\left(\max \left\{\omega_{b}(\Theta(a, c), \Theta(l, m)), \omega_{b}(\Theta(c, a), \Theta(m, l))\right\},\right. \\
& \left.L \max \left\{\omega_{b}(\Theta(a, c), \Theta(l, m)), \omega_{b}(\Theta(c, a), \Theta(m, l))\right\}\right),
\end{aligned}
$$

for all $a, c, l, m \in \Upsilon, \alpha>0$ with $\Theta(a, c) \preceq \Theta(l, m)$ and $\Theta(c, a) \succeq \Theta(m, l)$ or $\Theta(c, a) \preceq$ $\Theta(m, l)$ and $\Theta(a, c) \succeq \Theta(l, m)$. Then, $\Xi$ and $\Theta$ have a coupled coincidence point in $\Upsilon$.

Definition 12 [21] Let $(\Upsilon, \preceq)$ be a partially ordered set, $\Xi: \Upsilon \times \Upsilon \rightarrow \Upsilon$ and $\Theta: \Upsilon \rightarrow$ $\Upsilon$. We say that $\Xi$ is $\Theta$-increasing with respect to $\preceq$ iffor any $a, c \in \Upsilon$

$\Theta a_{1} \preceq \Theta a_{2}$ implies $\Xi\left(a_{1}, c\right) \preceq \Xi\left(a_{2}, c\right)$ and $\Theta c_{1} \preceq \Theta c_{2}$ implies $\Xi\left(a, c_{1}\right) \preceq \Xi\left(a, c_{2}\right)$.

Taking in to account the above definition, we obtain the following result:

Corollary 4 Let $\left(\Upsilon, \preceq, \omega_{b}\right)$ be a RPOCbML space (with parameter $s>1$ ). Assume that $\Xi: \Upsilon \times \Upsilon \rightarrow \Upsilon$ and $\Theta: \Upsilon \rightarrow \Upsilon$ are two mappings such that the following conditions are verified:

(1) $\Xi(\Upsilon \times \Upsilon) \subseteq \theta(\Upsilon)$.

(2) $\Theta(\Upsilon)$ is closed.

(3) $\Xi$ is $\Theta$-increasing with respect to $\preceq$. 
(4) There exist two elements $a_{0}, c_{0} \in \Upsilon$ such that $\Theta a_{0} \preceq \Xi\left(a_{0}, c_{0}\right)$ and $\theta c_{0} \succeq \Xi\left(c_{0}, a_{0}\right)$. Suppose that there exist $\psi \in \Psi, 0<L<1$ and $f \in C$ such that:

$$
\begin{array}{r}
\psi\left(s^{\alpha} \omega_{b}(\Xi(a, c), \Xi(l, m))\right) \leq f\left(\psi\left(\max \left\{\omega_{b}(\Theta a, \Theta l), \omega_{b}(\Theta c, \Theta m)\right\}\right),\right. \\
\left.L \max \left\{\omega_{b}(\Theta a, \Theta l), \omega_{b}(\Theta c, \Theta m)\right\}\right),
\end{array}
$$

for all $a, c, l, m \in \Upsilon, \alpha>0$ with $\Theta a \preceq \Theta l$ and $\Theta c \succeq \Theta m$ or $\Theta c \preceq \Theta m$ and $\Theta a \succeq \Theta l$. Then, $\Xi$ and $\Theta$ have a coupled coincidence point in $\Upsilon$.

Definition 13 [17] Let $(\Upsilon, \preceq)$ be a partially ordered set, $\Xi: \Upsilon \times \Upsilon \rightarrow \Upsilon$. We say that $\Xi$ is increasing with respect to $\preceq$ if for any $a, c \in \Upsilon$

$$
a_{1} \preceq a_{2} \text { implies } \Xi\left(a_{1}, c\right) \preceq \Xi\left(a_{2}, c\right) \text { and } c_{1} \preceq c_{2} \text { implies } \Xi\left(a, c_{1}\right) \preceq \Xi\left(a, c_{2}\right) \text {. }
$$

According to the above definition, we have the following corollary:

Corollary 5 Let $\left(\Upsilon, \preceq, \omega_{b}\right)$ be a RPOCbML space (with parameter $\left.s>1\right)$. Assume that $\Xi: \Upsilon \times \Upsilon \rightarrow \Upsilon$ is mapping such that the following conditions are justified:

(1) $\Xi$ is increasing with respect to $\preceq$.

(2) There exist two elements $a_{0}, c_{0} \in X$ such that $a_{0} \preceq \Xi\left(a_{0}, c_{0}\right)$ and $c_{0} \succeq \Xi\left(c_{0}, a_{0}\right)$. Suppose that there exist $\psi \in \Psi, 0<L<1$ and $f \in C$ such that:

$$
\begin{array}{r}
\psi\left(s^{\alpha} \omega_{b}(\Xi(a, c), \Xi(l, m))\right) \leq f\left(\psi\left(\max \left\{\omega_{b}(a, l), \omega_{b}(c, m)\right\}\right),\right. \\
\left.L \max \left\{\omega_{b}(a, l), \omega_{b}(c, m)\right\}\right),
\end{array}
$$

for all $a, c, l, m \in \Upsilon, \alpha>0$ with $a \preceq l$ and $c \succeq m$ or $c \preceq m$ and $a \geq l$. Then, $\Xi$ has $a$ coupled fixed point in $\Upsilon$.

\section{Supportive application}

Recently fixed point theory has expanded to its importance in many applications including finding analytical solutions for differential and integral equations (see [22-24] and references therein). In fact, this section is the most important part of the paper, where we shall study the existence of a unique solution to a non-linear integral equation using the theoretical results presented in the previous section.

Let $\Pi$ denote the class functions $\pi:[0, \infty) \rightarrow[0, \infty)$ such that $\pi$ is increasing and there exists $\psi \in \Psi, 0<L<1$, and $f \in \complement$ such that $\pi(\kappa)=\frac{1}{4} f(\psi(\kappa), L \kappa)$ for all $\kappa \in[0, \infty)$. Suppose the following problem:

$$
a(t)=\int_{p}^{q}\left(K_{1}(t, \rho)+K_{2}(t, \rho)\right)\left(\frac{g(\rho, a(\rho))+h(\rho, a(\rho))}{1+g(\rho, a(\rho))+h(\rho, a(\rho)}\right) d \rho,
$$

for all $t \in[p, q]$. Suppose that $K_{1}, K_{2}, g, h$, satisfies the following hypotheses:

(i) For all $t, \rho \in[p, q], K_{1}(t, \rho) \geq 0$ and $K_{2}(t, \rho) \geq 0$;

(ii) For all $a, c \in \mathbb{R}$ such that $a \geq c$, there exist $\sigma, \varsigma$ such that

$$
\begin{aligned}
& 0 \leq g(\rho, a)+g(\rho, c) \leq \sigma \pi(a+c), \\
& 0 \leq h(\rho, a)+h(\rho, c) \leq \varsigma \pi(a+c)
\end{aligned}
$$


(iii) We get

$$
\max \left\{\sigma^{2}, \varsigma^{2}\right\}\left\{\sup _{t \in[a, b]} \int_{p}^{q}\left(K_{1}(t, \rho)+K_{2}(t, \rho)\right) d \rho\right\}^{2} \leq 1 ;
$$

(iv) For all $t \in[p, q]$, there exist continuous functions $\varpi, \xi:[p, q] \rightarrow \mathbb{R}$ such that

$$
\begin{aligned}
\varpi(t) \leq & \int_{p}^{q} K_{1}(t, \rho)\left(\frac{g(\rho, \varpi(\rho))+h(\rho, \xi(\rho))}{1+g(\rho, \xi(\rho))+h(\rho, \varpi(\rho))}\right) d \rho \\
& +\int_{p}^{q} K_{2}(t, \rho)\left(\frac{g(\rho, \xi(\rho))+h(\rho, \varpi(\rho))}{1+g(\rho, \varpi(\rho))+h(\rho, \xi(\rho))}\right) d \rho,
\end{aligned}
$$

and

$$
\begin{aligned}
\xi(t) \geq & \int_{p}^{q} K_{1}(t, \rho)\left(\frac{g(\rho, \xi(\rho))+h(\rho, \varpi(\rho))}{1+g(\rho, \varpi(\rho))+h(\rho, \xi(\rho))}\right) d \rho \\
& +\int_{p}^{q} K_{2}(t, \rho)\left(\frac{g(\rho, \varpi(\rho))+h(\rho, \xi(\rho))}{1+g(\rho, \xi(\rho))+h(\rho, \varpi(\rho))}\right) d \rho .
\end{aligned}
$$

Now, we formulate the theorem concerning the existence of a unique solution of a nonlinear integral equation (16) as follows:

Theorem 3 Suppose that the problem (16) with $K_{1}, K_{2} \in C([p, q] \times[p, q], \mathbb{R})$ and $g, h \in C([p, q] \times \mathbb{R} \times \mathbb{R})$, then the problem (16) has a solution in $C([p, q], \mathbb{R})$, provided that hypotheses (i)-(iv) are verified.

Proof Assume that $\Upsilon=C([a, b], \mathbb{R})$ is the set of real continuous functions on $[p, q]$ equipped with

$$
\omega_{b}(a, c)=\sup _{t \in[p, q]}(|a(t)+c(t)|)^{2} \text { for all } a, c \in \Upsilon .
$$

Thus, the pair $\left(\Upsilon, \omega_{b}\right)$ is a complete $b$-metric-like space with a coefficient $s=2$, and $\left(\Upsilon, \omega_{b}, \preceq\right)$ is a RPOCbML space if $a \preceq c$ whenever $a(t) \preceq c(t)$ for all $t \in[p, q]$. Let the sequence of monotone non-decreasing $\left\{a_{n}\right\}$ in $\Upsilon$ such that $\lim _{n \rightarrow \infty} a_{n}=a \in \Upsilon$, then the sequences (sequences of real numbers)

$$
a(t) \leq a_{2}(t) \leq \ldots \leq a_{n}(t) \leq \cdots
$$

converge also to $a(t)$ for all $t \in[p, q]$. So $a_{n}(t) \leq a(t)$ for all $t \in[p, q], n \in \mathbb{N}$. Therefore, $a_{n} \preceq a$ for all $n$. By the same manner, one can show that the monotone non-increasing sequence $\left\{c_{n}\right\}$ in $\Upsilon$ such that $\lim _{n \rightarrow \infty} c_{n}=c \in \Upsilon$ is a lower bound for all the elements in the sequence, i.e., $c \preceq c_{n}$ for all $n$. Therefore, the regularity condition hold.

Also, if we define an order relation on $\Upsilon \times \Upsilon$ as $a \preceq l, c \succeq m$ for all $a, c, l, m \in \Upsilon$, we conclude that $\Upsilon \times \Upsilon$ is a partially ordered set. 
Define the mapping $\Xi: \Upsilon \times \Upsilon \rightarrow \Upsilon$ by

$$
\begin{aligned}
\Xi(a, c)(t)= & \int_{p}^{q} K_{1}(t, \rho)\left(\frac{g(\rho, a(\rho))+h(\rho, c(\rho))}{1+g(\rho, c(\rho))+h(\rho, a(\rho))}\right) d \rho \\
& +\int_{p}^{q} K_{2}(t, \rho)\left(\frac{g(\rho, c(\rho))+h(\rho, a(\rho))}{1+g(\rho, a(\rho))+h(\rho, c(\rho))}\right) d \rho,
\end{aligned}
$$

for all $t \in[p, q]$. It is clear that if the mapping $\Xi$ has a coupled fixed point in $\Upsilon=$ $C([p, q], \mathbb{R})$, then it is a solution of the problem $(16)$.

Now, we shall prove that the mapping $\Xi$ is increasing with $a_{1} \preceq a_{2}$, so, $a_{1}(t) \leq a_{2}(t)$ for all $t \in[p, q]$; we can write

$$
\begin{aligned}
& \Xi\left(a_{1}, c\right)(t)-\Xi\left(a_{2}, c\right)(t) \\
= & \int_{p}^{q} K_{1}(t, \rho)\left(\frac{g\left(\rho, a_{1}(\rho)\right)+h(\rho, c(\rho))}{1+g(\rho, c(\rho))+h\left(\rho, a_{1}(\rho)\right)}\right) d \rho+\int_{p}^{q} K_{2}(t, \rho)\left(\frac{g(\rho, c(\rho))+h\left(\rho, a_{1}(\rho)\right)}{1+g(\rho, a(\rho))+h(\rho, c(\rho))}\right) d \rho \\
& -\int_{p}^{q} K_{1}(t, \rho)\left(\frac{g\left(\rho, a_{2}(\rho)\right)+h(\rho, c(\rho))}{1+g(\rho, c(\rho))+h\left(\rho, a_{2}(\rho)\right)}\right) d \rho-\int_{p}^{q} K_{2}(t, \rho)\left(\frac{g(\rho, c(\rho))+h\left(\rho, a_{2}(\rho)\right)}{1+g\left(\rho, a_{2}(\rho)\right)+h(\rho, c(\rho))}\right) d \rho \\
\leq & \int_{p}^{q} K_{1}(t, \rho)\left(g\left(\rho, a_{1}(\rho)\right)+h(\rho, c(\rho))\right) d \rho+\int_{p}^{q} K_{2}(t, \rho)\left(g(\rho, c(\rho))+h\left(\rho, a_{1}(\rho)\right)\right) d \rho \\
& -\int_{p}^{q} K_{1}(t, \rho)\left(g\left(\rho, a_{2}(\rho)\right)+h(\rho, c(\rho))\right) d \rho-\int_{p}^{q} K_{2}(t, \rho)\left(g(\rho, c(\rho))+h\left(\rho, a_{2}(\rho)\right)\right) d \rho \\
= & \int_{p}^{q} K_{1}(t, \rho)\left(g\left(\rho, a_{1}(\rho)\right)-g\left(\rho, a_{2}(\rho)\right)\right) d \rho+\int_{p}^{q} K_{2}(t, \rho)\left(h\left(\rho, a_{1}(\rho)\right)-h\left(\rho, a_{2}(\rho)\right)\right) d \rho \leq 0 .
\end{aligned}
$$

Therefore, $\Xi\left(a_{1}, c\right)(t) \leq \Xi\left(a_{2}, c\right)(t)$ for all $t \in[p, q]$. Hence, $\Xi\left(a_{1}, c\right) \preceq \Xi\left(a_{2}, c\right)$. Similarly, if $c_{1} \preceq c_{2}$, so $c_{1}(t) \leq c_{2}(t)$ for all $t \in[p, q]$, we have

$$
\begin{aligned}
& \Xi\left(a, c_{1}\right)(t)-\Xi\left(a, c_{2}\right)(t) \\
= & \int_{p}^{q} K_{1}(t, \rho)\left(\frac{g(\rho, a(\rho))+h\left(\rho, c_{1}(\rho)\right)}{1+g\left(\rho, c_{1}(\rho)\right)+h(\rho, a(\rho))}\right) d \rho+\int_{p}^{q} K_{2}(t, \rho)\left(\frac{g\left(\rho, c_{1}(\rho)\right)+h(\rho, a(\rho))}{1+g(\rho, a(\rho))+h\left(\rho, c_{1}(\rho)\right)}\right) d \rho \\
& -\int_{p}^{q} K_{1}(t, \rho)\left(\frac{g(\rho, a(\rho))+h\left(\rho, c_{2}(\rho)\right)}{1+g\left(\rho, c_{2}(\rho)\right)+h(\rho, a(\rho))}\right) d \rho-\int_{p}^{q} K_{2}(t, \rho)\left(\frac{g\left(\rho, c_{2}(\rho)\right)+h(\rho, a(\rho))}{1+g(\rho, a(\rho))+h\left(\rho, c_{2}(\rho)\right)}\right) d \rho \\
\leq & \int_{p}^{q} K_{1}(t, \rho)\left(g(\rho, a(\rho))+h\left(\rho, c_{1}(\rho)\right)\right) d \rho+\int_{p}^{q} K_{2}(t, \rho)\left(g\left(\rho, c_{1}(\rho)\right)+h(\rho, a(\rho))\right) d \rho \\
& -\int_{p}^{q} K_{1}(t, \rho)\left(g(\rho, a(\rho))+h\left(\rho, c_{2}(\rho)\right)\right) d \rho-\int_{p}^{q} K_{2}(t, \rho)\left(g\left(\rho, c_{2}(\rho)\right)+h(\rho, a(\rho))\right) d \rho \\
= & \int_{p}^{q} K_{1}(t, \rho)\left(h\left(\rho, c_{1}(\rho)\right)-h\left(\rho, c_{2}(\rho)\right)\right) d \rho+\int_{p}^{q} K_{2}(t, \rho)\left(g\left(\rho, c_{1}(\rho)\right)-g\left(\rho, c_{2}(\rho)\right)\right) d \rho \leq 0 .
\end{aligned}
$$

Therefore, $\Xi\left(a, c_{1}\right)(t) \leq \Xi\left(a, c_{2}\right)(t)$ for all $t \in[p, q]$. Hence, $\Xi\left(a, c_{1}\right) \preceq \Xi\left(a, c_{2}\right)$. From the above results, we deduce that the mapping $\Xi$ is increasing with respect to the variables $a, c$. 
Finally, we illustrate that the contraction condition of Corollary 5 hold, for all $a, c, l, m \in$ $\Upsilon$ such that $a \preceq l, m \preceq c$,

$$
\begin{aligned}
& \omega_{b}(\Xi(a, c), \Xi(l, m)) \\
& =\sup _{t \in[p, q]}(|\Xi(a, c)(t)+\Xi(l, m)(t)|)^{2} \\
& =\left\{\begin{array}{c}
\sup _{t \in[p, q]} \mid \int_{p}^{q} K_{1}(t, \rho)\left(\frac{g(\rho, a(\rho))+h(\rho, c(\rho))}{1+g(\rho, c(\rho))+h(\rho, a(\rho))}\right) d \rho+\int_{p}^{q} K_{2}(t, \rho)\left(\frac{g(\rho, c(\rho))+h(\rho, a(\rho))}{1+g(\rho, a(\rho))+h(\rho, c(\rho))}\right) d \rho \\
\quad+\int_{p}^{q} K_{1}(t, \rho)\left(\frac{g(\rho, l(\rho))+h(\rho, m(\rho))}{1+g(\rho, m(\rho))+h(\rho, l(\rho))}\right) d \rho+\int_{p}^{q} K_{2}(t, \rho)\left(\frac{g(\rho, m(\rho))+h(\rho, l(\rho))}{1+g(\rho, l(\rho))+h(\rho, m(\rho))}\right) d \rho \mid
\end{array}\right\}^{2} \\
& \leq\left\{\begin{array}{c}
\sup _{t \in[p, q]} \mid\left(\begin{array}{c}
\int_{p}^{q} K_{1}(t, \rho)(g(\rho, a(\rho))+h(\rho, c(\rho))) d \rho \\
q \\
+\int_{b}^{q} K_{2}(t, \rho)(g(\rho, c(\rho))+h(\rho, a(\rho))) d \rho
\end{array}\right) \\
+\left(\begin{array}{c}
\int_{p}^{q} K_{1}(t, \rho)(g(\rho, l(\rho))+h(\rho, m(\rho))) d \rho \\
+\int_{p}^{q} K_{2}(t, \rho)(g(\rho, m(\rho))+h(\rho, l(\rho))) d \rho
\end{array}\right) \mid
\end{array}\right\} \\
& =\left\{\sup _{t \in[p, q]} \mid\left(\begin{array}{c}
\mid \int_{p}^{q} K_{1}(t, \rho)[(g(\rho, a(\rho))+g(\rho, l(\rho)))+(h(\rho, c(\rho))+h(\rho, m(\rho)))] d \rho \\
+\int_{p}^{q} K_{2}(t, \rho)[(g(\rho, c(\rho))+g(\rho, m(\rho)))+(h(\rho, a(\rho))+h(\rho, l(\rho)))] d \rho \mid
\end{array}\right)\right\}^{2} .
\end{aligned}
$$

Applying condition (ii), we have

$$
\begin{aligned}
& \omega_{b}(\Xi(a, c), \Xi(l, m)) \\
\leq & \left\{\sup _{t \in[p, q]}\left(\begin{array}{c}
\mid \int_{p}^{q} K_{1}(t, \rho)[\sigma \pi(a(\rho)+l(\rho))+\varsigma \pi(c(\rho)+m(\rho))] d \rho \\
+\int_{p}^{q} K_{2}(t, \rho)[\sigma \pi(c(\rho)+m(\rho))+\varsigma \pi(a(\rho)+l(\rho))] d \rho \mid
\end{array}\right)\right\}^{2} \\
\leq & \max \left\{\sigma^{2}, \varsigma^{2}\right\}\left\{\sup _{t \in[p, q]}\left(\mid \int_{p}^{q}\left[K_{1}(t, \rho)+K_{2}(t, \rho)\right][\pi(|a(\rho)+l(\rho)|)+\pi(|c(\rho)+m(\rho)|)] d \rho\right)\right\}^{2} .
\end{aligned}
$$

By the definition of $\pi$ and the distance $\omega_{b}$, we can write

$$
\pi|a(\rho)+l(\rho)|^{2} \leq \pi \omega_{b}(a, l) \text { and } \pi|c(\rho)+m(\rho)|^{2} \leq \pi \omega_{b}(c, m) \text { for all } \rho \in[p, q] .
$$

It follows from (17), (18), and condition (iii) that

$$
\begin{aligned}
& \omega_{b}(\Xi(a, c), \Xi(l, m)) \\
\leq & \max \left\{\sigma^{2}, \varsigma^{2}\right\} \times\left[\pi^{2} \omega_{b}(a, l)+\pi^{2} \omega_{b}(c, m)\right] \times\left\{\sup _{t \in[p, q]} \int_{p}^{q}\left(K_{1}(t, \rho)+K_{2}(t, \rho)\right) d \rho\right\}^{2} \\
\leq & \pi^{2} \omega_{b}(a, l)+\pi^{2} \omega_{b}(c, m) \\
\leq & \pi^{2}\left(\omega_{b}(a, l)+\omega_{b}(c, m)\right) \\
\leq & 2 \pi^{2}\left(\max \left\{\omega_{b}(a, l), \omega_{b}(c, m)\right\}\right) \text { since } i+j \leq 2 \max \{i, j\} \text { for all } i, j \in \mathbb{R} \\
\leq & 2 \times \frac{1}{16} f\left(\psi\left(\max \left\{\omega_{b}(a, l), \omega_{b}(c, m)\right\}\right), L \max \left\{\omega_{b}(a, l), \omega_{b}(c, m)\right\}\right) \\
= & \frac{1}{8} f\left(\psi\left(\max \left\{\omega_{b}(a, l), \omega_{b}(c, m)\right\}\right), L \max \left\{\omega_{b}(a, l), \omega_{b}(c, m)\right\}\right) .
\end{aligned}
$$


Thus, for $a \preceq l, m \preceq c$, we obtain that

$$
2^{3} \omega_{b}(\Xi(a, c), \Xi(l, m)) \leq f\left(\psi\left(\max \left\{\omega_{b}(a, l), \omega_{b}(c, m)\right\}\right), L \max \left\{\omega_{b}(a, l), \omega_{b}(c, m)\right\}\right),
$$

taking $\psi \in \Psi$ such that $\psi(\kappa)=\kappa$, for all $\kappa \in[0, \infty]$ with $s=2$ and $\alpha=3$, we have

$$
\psi\left(s^{\alpha} \omega_{b}(\Xi(a, c), \Xi(l, m))\right) \leq f\left(\psi\left(\max \left\{\omega_{b}(a, l), \omega_{b}(c, m)\right\}\right), L \max \left\{\omega_{b}(a, l), \omega_{b}(c, m)\right\}\right) .
$$

Also, condition (iv) leads to $\varpi(t) \leq \Xi(\varpi, \xi)(t)$ and $\xi(t) \geq \Xi(\xi, \varpi)(t)$ for all $t \in[p, q]$, that is $\varpi \preceq \Xi(\varpi, \xi)$ and $\xi \succeq \Xi(\xi, \varpi)$. Therefore, all the hypothesis requirements of Corollary 5 are satisfied. Then, the mapping $\Xi$ has a coupled fixed point which is a solution of the problem $(16)$ in $\Upsilon=C([p, q], \mathbb{R})$.

\section{Conclusion}

Recently, the technique of coincidence point has become one of the important issues that contribute to finding the analytical solution of integral equations, which in turn facilitates the numerical solution. Therefore, many researchers are interested in this trend so that the differential equation can be converted to their integral counterparts and then easily solved. This paper is the same line, where we discussed some common coincidence points for nonlinear mappings under the class of a RPOCbML space; also, we proceed with two examples to support theoretical results and analytical solution for the nonlinear integral equation illustrated.

\section{Acknowledgements}

Not applicable

\section{Authors' contributions}

$\mathrm{HAH}$ wrote the title, abstract, examples, application, and conclusion and fixed many language errors. DMA wrote the theoretical work, introduction, and references. RAR fixed some spelling errors and gave some important sentences to support the paper. All authors read and approved the final manuscript.

\section{Funding}

There is no sponsor.

\section{Availability of data and materials}

Data sharing not applicable to this article as no datasets were generated or analyzed during the current study.

\section{Competing interests}

The authors declare that they have no competing interests.

\section{Author details}

${ }^{1}$ Department of Mathematics, Faculty of Science, Sohag University, Sohag 82524, Egypt. ${ }^{2}$ Department of Mathematics, Faculty of Education, Sanaa University, Sanaa 1247, Yemen. ${ }^{3}$ Department of Mathematics, Faculty of Science, Assuit University, Assuit 71516, Egypt.

Received: 18 October 2019 Accepted: 18 December 2019

Published online: 29 January 2020

\section{References}

1. Banach, S.: Sur les opérations dans les ensembles abstraits et leur application aux équations intégrales. Fund. Math. 3, 133-181 (1922)

2. Matthews, S. G.: Partial metric topology in: Proc. 8th Summer Conference on General Topology and Applications. Ann. New York Acad. Sci. 728, 183-197 (1994)

3. Bakhtin, I. A.: The contraction principle in quasimetric spaces. Funct. Anal. 30, 26-37 (1989)

4. Czerwik, S.: Contraction mappings in b-metric spaces. Acta Math. Inf. Univ. Ostrav. 1, 5-11 (1993)

5. Shah, M., Simic, S., Hussain, N., Sretenovic, A., Radenovic, S.: Common fixed points theorems for occasionally weakly compatible pairs on cone metric type spaces. J. Comput. Anal. Appl. 14, 290-297 (2012)

6. Hussain, N., Shah, M.: KKM mappings in cone b-metric spaces. Comput. Math. Appl. 62, 1677-1684 (2011)

7. Khamsi, M., Hussain, N.: KKM mappings in metric type spaces. Nonlinear Anal. 73(2010), 3123-3129

8. Radenović, S., Zoto, K., Dedović, N., Šešum-Cavic, V., Ansari, A.: Bhaskar-Guo-Lakshmikantam-Ćirić type results via new functions with a pplications to integral equations. Appl. Math. Comput. (2019). Artical in press. https://doi.org/10. 1016/j.amc.2019.03.057 
9. Amini-Harandi, A.: Metric-like spaces, partial metric spaces and fixed points. Fixed Point Theory Appl. 2012, 204 (2012). https://doi.org/10.1186/1687-1812-2012-204

10. Alghamdi, M. A., Hussain, N., Salimi, P.: Fixed point and coupled fixed point theorems on b-metric-like spaces. J. Inequalities Appl. 402(2013), 1-25 (2013)

11. Hussain, N., Roshan, J. R., Parvaneh, V., Kadelburg, Z.: Fixed points of contractive mappings in b-metric-like spaces. Sci. World J., 1-15 (2014). https://doi.org/10.1155/2014/471827

12. Aydi, A., Felhi, A., Sahmim, S.: Common fixed points via implicit contractions on b-metric-like spaces. J. Nonlinear Sci. Appl. 10, 1524-1537 (2017)

13. Nashine, H. K., Kadelburg, Z:. Existence of solutions of Cantilever Beam Problem via $\alpha-\beta-F G-$ contractions in b-metric-like spaces. Filomat. 31, 3057-3074 (2017)

14. Hammad, H. A., De la Sen, M.: Generalized contractive mappings and related results in b-metric like spaces with an application. Symmetry. 11(667) (2019). https://doi.org/10.3390/sym11050667

15. Hammad, H. A., De la, Sen, M.: Solution of nonlinear integral equation via fixed point of cyclic $\alpha_{L}^{\psi}-$ rational contraction mappings in metric-like spaces. Bull. Braz. Math. Soc. New Ser. 347 (2019). https://doi.org/10.1007/ s00574-019-00144-1

16. Guo, D., Lakshmikantham, V.: Coupled fixed points of nonlinear operators with applications. Nonlinear Anal. 11, 623-632 (1987)

17. Bhaskar, T. G., Lakshmikantham, V.: Fixed point theorems in partially ordered metric spaces and applications. Nonlinear Anal. Theor. 65, 1379-1393 (2006)

18. Lakshmikantham, V., Ćirić, L. j. B.: Coupled random fixed point theorems for nonlinear contractions in partially ordered metric spaces. Stoch. Anal. Appl. 27, 1246-1259 (2009)

19. Hussain, N., Abbas, M., Azam, A., Ahmad, J.: Coupled coincidence point results for a generalized compatible pair with applications. Fixed Point Theory Appl. 62 (2014). https://doi.org/10.1186/1687-1812-2014-62

20. Ansari, A. H., Chandok, S., lonescu, C.: Fixed point theorems on b-metric spaces for weak contractions with auxiliary functions. J. Inequal. Appl. 429, 318 (2014)

21. Lakshmikantham, V., Ćirić, L. j. B.: Coupled fixed point theorems for nonlinear contractions in partially ordered metric spaces. Nonlinear Anal. Theory Methods Appl. 70, 4341-4349 (2009)

22. Radenović, S.: Coupled fixed point theorems for monotone mappings in partially ordered metric spaces. Krag. J. Math. 38(2), 249-257 (2014)

23. Hammad, H. A., De la, Sen, M.: A solution of Fredholm integral equation by using the cyclic $\eta_{q}^{s}$-rational contractive mappings technique in b-metric-like spaces. Symmetry. 11(11) (2019). https://doi.org/10.3390/sym11091184

24. Hammad, H. A., De la, Sen, M.: A coupled fixed point technique for solving coupled systems of functional and nonlinear integral equations. Mathematics. 7(634) (2019). https://doi.org/10.3390/math7070634

\section{Publisher's Note}

Springer Nature remains neutral with regard to jurisdictional claims in published maps and institutional affiliations.

\section{Submit your manuscript to a SpringerOpen ${ }^{\circ}$ journal and benefit from:}

- Convenient online submission

Rigorous peer review

- Open access: articles freely available online

- High visibility within the field

- Retaining the copyright to your article

Submit your next manuscript at $\boldsymbol{\triangleright}$ springeropen.com 